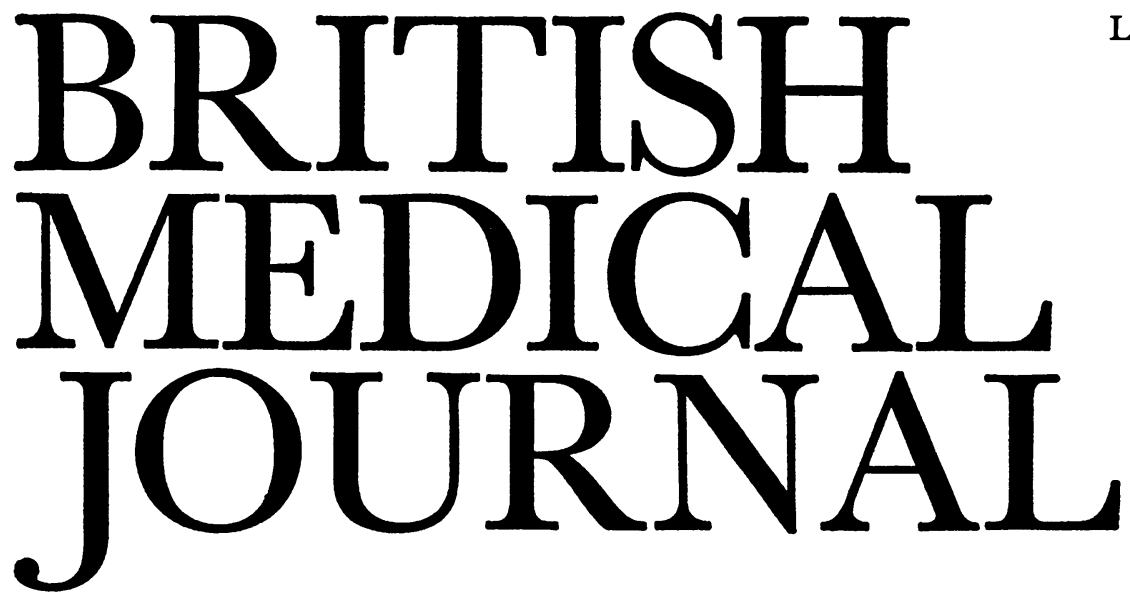

\title{
Intermediate Coronary Syndrome
}

Cardiologists have a reputation for coining new terms in which to obscure previously well recognized entities. When angina pectoris recurs at rest and infarction seems imminent, or angina is of recent onset or has newly become severe or prolonged, the clinical syndromes have been given many names. These included acute coronary insufficiency, ${ }^{1-5}$ the intermediate coronary syndrome, ${ }^{67}$ acute myocardial ischaemia, ${ }^{89}$ pre-infarction angina, ${ }^{10-15}$ impending myocardial infarction, ${ }^{16}$ status anginosus, ${ }^{17}$ unstable angina, ${ }^{18-20}$ and accelerated angina. ${ }^{21}$

Nearly $20 \%$ of admissions to coronary care units are subsequently placed in this intermediate category between stable angina and acute infarction. ${ }^{5}$ Reports vary on the risk of the patient's progressing to infarction from this unstable state. Earlier estimates were high-22\% within two months, ${ }^{2}$ $41 \%$ within three months, ${ }^{11} 29 \%$ within six months. ${ }^{16} \mathrm{Be}-$ cause of these alarming figures the operation of myocardial revascularization by aorto-coronary bypass grafting after emergency coronary angiography has recently been undertaken in the hope of averting the impending infarct. 91319

Recent studies have shown a lower risk of progression to infarction during the acute phase. A study of unstable angina (with a rather broad definition) carried out in Edinburgh gave an infarction risk of $14 \%$ and a mortality of under $2 \% .{ }^{20}$ Previous reports have also shown that the immediate outlook for these patients when treated medically is fairly good, and the early mortality has been under $6 \% .137$ These figures do not seem to justify the risk of coronary angiography and surgery in the acute phases under conditions of stress and urgency.

The usefulness of beta-adrenegic receptor blocking drugs during this acute phase has recently become established. ${ }^{12}$ Before their advent treatment was symptomatic and empirical. Active treatment was expected, and anticoagulants, particularly intravenous heparin, were considered obligatory. ${ }^{2}$ The rationale of using these drugs is now doubtful and conflicts with modern observations on the low incidence of coronary thrombosis in myocardial infarction. Furthermore anticoagulants fail to influence the development of arterial thrombosis or to accelerate thrombolysis. They are therefore irrelevant to the management of the intermediate coronary syndrome.

Writing under this title, S. J. Fischel and his colleagues from Boston, U.S.A., have related the therapy of the syndrome to the angiographic appearances. ${ }^{22}$ The Boston workers had stringent criteria for the diagnosis of the intermediate coronary syndrome. These were recurring angina at rest with single attacks lasting more than 20 minutes, incomplete relief of pain with nitrates, an unstable electrocardiogram with fluctuating changes in the ST and $\mathrm{T}$ waves, but normal or non-diagnostic (less than $40 \%$ above normal) levels of serum enzymes. Twenty-three patients were studied, and 21 of these were investigated by coronary angiography. Oral propranolol $20 \mathrm{mg}$ 4-hourly was given, and the dose was rapidly increased until either pain was controlled or the heart rate remained consistently below 60 per minute. Pain was relieved quickly in 17 out of 20 patients treated in this way. Beta-adrenergic blockade was associated with reduction in the mean heart rate from 90 to 64 per minute and in systolic blood pressure from 159 to $113 \mathrm{~mm} \mathrm{Hg}$ in treated patients. Before treatment the pulse rate and blood pressure had risen during attacks of anginal pain, and clinical signs of left ventricular failure had developed in 27 patients but resolved within 24 hours of starting propranolol. Only three patients did not respond, and they were treated surgically during the acute phase.

Coronary angiograms showed coronary artery disease whose appearance did not differ in severity, type, or distribution from that in patients with chronic stable angina except that there were few collateral vessels. One patient had normal coronary arteries. This surprising observation in patients with typical angina has been made repeatedly in most cardiac centres. Patients with normal coronary arteries can form a high proportion of the patients referred for investigation of chest pain, reaching a quarter of the total in one series. ${ }^{23}$ These patients do not have small vessel disease or other recognizable lesions and usually do well. The origin of their pain is probably outside the heart in most cases even when it resembles the pain of the intermediate coronary syndrome, but a few may have recurrent pericardial pain following previously unrecognized pericarditis. The Prinzmetal variant of angina, in which pain is associated with elevation rather than the usual depression of the ST segment on the electrocardiogram, has been described in association with a normal coronary tree as well as in patients with occlusion of a single main vessel. Prinzmetal's angina is exceptional in 
carrying a high risk of ventricular fibrillation regardless of the coronary anatomy and remains ill-understood.

Spontaneously occurring angina is preceded by a rise in heart rate and blood pressure, ${ }^{24}$ which augment myocardial oxygen demands. It is associated with a hypoxia-induced reduction in ejector efficiency from the left ventricule, ${ }^{25}$ which may further intensify ischaemia. The benefit of propranolol can be related to counteraction of these provocations, particularly when recurring pain further increases the rate of sympathetic stimulation. This clinical benefit from propranolol, even despite left ventricular failure (when it might be thought contraindicated) is worth stressing. When left ventricular dysfunction is associated with painful ischaemia (rather than infarction), ventricular efficiency is regained when the ischaemia is relieved. The Boston workers emphasize the use of beta-adrenergic blockade to stabilize the patient, thereby enabling the majority to be investigated and if necessary treated surgically after readjustment and loss of pain at rest. Fourteen patients were subsequently operated on and 12 benefited. There were no deaths in their series during the acute phase, but three medically treated patients died later.

Though these acute coronary syndromes are not usually precursors of imminent infarction or death, they may represent a phase in the course of progressive coronary artery disease before the collateral supply to an obstructed coronary segment becomes adequate. ${ }^{22}$ Propranolol is rapidly effective in relieving pain and carries less risk than emergency surgery. After being tided over the acute phase by medical means, investigation and bypass surgery may be followed by early resumption of normal activity, with the hope also of reducing the risk of myocardial infarction in the following months.

${ }^{1}$ Littman, D., and Baur, J. H., jun., Circulation, 1952, 5, 189.

2 Wood, P., British Medical fournal, 1961, 1, 1779.

3 Murnaghan, D., and Hickey, N., British Heart fournal, 1970, 32, 555.

4 Nichol, E. S., Phillips, W. C., and Casten, C., Annals of Internal Medicine,

$1959,50,1158$.
5 1972,45 , supp. 1,66 .

6 Graybiel, A., United States Armed Forces Medical fournal, 1955, 6, 1.

7 Graybiel, A., United States Armed Forces

8 Goble, A. J., and O'Brien, E. N., Lancet, 1958, 2, 873.

9 Chatterjee, K., et al., New England fournal of Medicine, 1972, 286, 1117.

10 Resiuk, W. H., American Heart fournal, 1962, 63, 290.

11 Vakil, R. J., American fournal of Cardiology, 1964, 14, 55.

12 Papazoglor, N. M., Circulation, 1971, 44, 303.

13 Vogel, J. H. K., et al., Chest, 1971, 59, 606.

14 Gazes, P. C., Mobley, E. M., and Faris, H. M., Circulation, 1972, supp. 2, Gazes,

15 Linhart, J. W., Beller, B. M., and Talley, R. C., Chest, 1972, 61, 312.

16 Beamish, R. E., and Storrie, V. M., Circulation, 1960, 21, 1107.

17 Harrison, T. R., and Reeves, T. J., in Principles and Problems of Ischemic Heart Disease, pp. 259-266. Year Book Medical Publishers, Chicago, 1968.

18 The Prodromal Symptoms of Myocardial Infarction with Sudden Death, W.H.O. Regional Office for Europe, Copenhagen, 1971

19 Conti, C. R., et al., Circulation, 1971, 44, supp. 2, 154

20 Fulton, M., et al., Lancet, 1972, 1, 860.

21 Scalon, P. J., et al., Circulation, 1973, 47, 19.

22 Fishl, S. J., Herman, M. V., and Gortun, R., New England fournal of Medicine, 1973, 288, 1193.

${ }^{23}$ Proudfit, W. L., Shirey, E. K., and Jones, F. M. jun., Circulation, 1966, 33, 901 .

${ }^{24}$ Robinson, B. F., Circulation, 1967, 35, 1073.

25 Amsterdam, E. A., et al., Clinical Research, 1969, 17, 225.

\section{Alcoholism Research}

Over 8,000 cases of alcoholism are being admitted to mental hospitals in England and Wales every year. ${ }^{1}$ Some go to highly specialized units. ${ }^{2}$ Others, and especially the vagrants, are admitted to the general wards of mental hospitals. ${ }^{3}$ No matter what the treatment setting, a familiar question then confronts the clinician: For how many weeks should his patient be asked to stay in hospital? The conventional wisdom today would probably suggest that a stay of two or three months is preferable to any shorter period. ${ }^{4-7}$ But we have little objective evidence on an issue that is as important to the individual patient as to national planning of services.

Recently P. J. A. Willems and colleagues ${ }^{8}$ described a controlled trial in which alcoholics were randomly allocated either to a short-stay inpatient regimen ( 31 patients, mean stay 20 days) or to a long-stay regimen (38 patients, mean stay 82 days), with follow-up of both groups for two years after discharge. This study represents something of a landmark in research on alcoholism.

Trials of different ways of treating alcoholism set special difficulties. Alcoholic patients are often hard to trace, and that Willems and his colleagues succeeded in tracing all but two of their 69 patients speaks of perseverance. Furthermore, the information obtained from the alcoholic himself is often suspect. The present report is based in every instance on the report of corroborative independent witnesses as wel! as self-report. On the basis of this painstaking piece of work the authors offer a conclusion which is boldly unequivocal: "Inpatient treatment for longer than one month is not necessary for the successful treatment of alcoholism."

What price now the accepted wisdom? Is the conclusion really so "clear" that any psychiatrist who continues to run his unit on the conventional model of two to three months' treatment must be seen as needlessly overtreating his patients, and this to much public cost? A critical reading of the present research might suggest that the conclusions which can fairly be drawn from the results are not altogether so certain as the authors claim. With such complex problems a controlled trial is perhaps seldom so sharp a scientific instrument as might be hoped. The research design in the present study involved, for instance, the giving of more intensive aftercare to the short-stay group than to the longstay group. Assessment was not "blind." Extrapolation from the present findings to the treatment given in other hospitals is to an extent limited by the authors' admission that the type of group therapy they offered was largely didactic or directed to immediate life problems. It could be argued that, when the group treatment regimen is directed to less superficial ends and utilizes more complex dynamic techniques, the longer stay is still likely to be preferable.

In an accompanying paper Willems and his colleagues ${ }^{10}$ describe some rating scales which can serve as prognostic indicators for the outcome of treatment. Their findings that higher scores on measures of impaired social stability (e.g., homelessness, job instability, criminal activity) all predict a bad outcome is fully in line with previous research. ${ }^{4}$ The practical inference might be that if better results are to be obtained with this type of patient imaginative experiment in aftercare and hostel accommodation is needed to repair the social rootlessness. 11 The finding that the degree of "insight" at discharge (but not at admission) is a strong indicator of a good outcome suggests by inference that inpatient treatment can certainly bring beneficial changes.

These two papers offer important further leads for study. Sadly, however, Governmental policies for research into alcoholism are so little integrated that a systematic followthrough of the present or any other research is in practice likely to remain a pious hope. Research on the prevention of alcoholism by the same sad token appears to be virtually non-existent. The growing problems of alcoholism in Britain deserve more than a haphazard research policy. 